\title{
Erratum: Machine learning for metallurgy III: A neural network potential for Al-Mg-Si [Phys. Rev. Materials 5, 053805 (2021)]
}

\author{
Abhinav C. P. Jain (, Daniel Marchand, Albert Glensk, M. Ceriotti, and W. A. Curtin
}

(Received 7 June 2021; published 9 July 2021)

DOI: 10.1103/PhysRevMaterials.5.079901

Figure 1 shows a corrected version of Fig. 9 in the original paper. Due to a plotting error, the energies of the original Fig. 9 were presented out of order. This figure shows all the values in the correct order. This new figure does not alter the conclusions of the original paper.

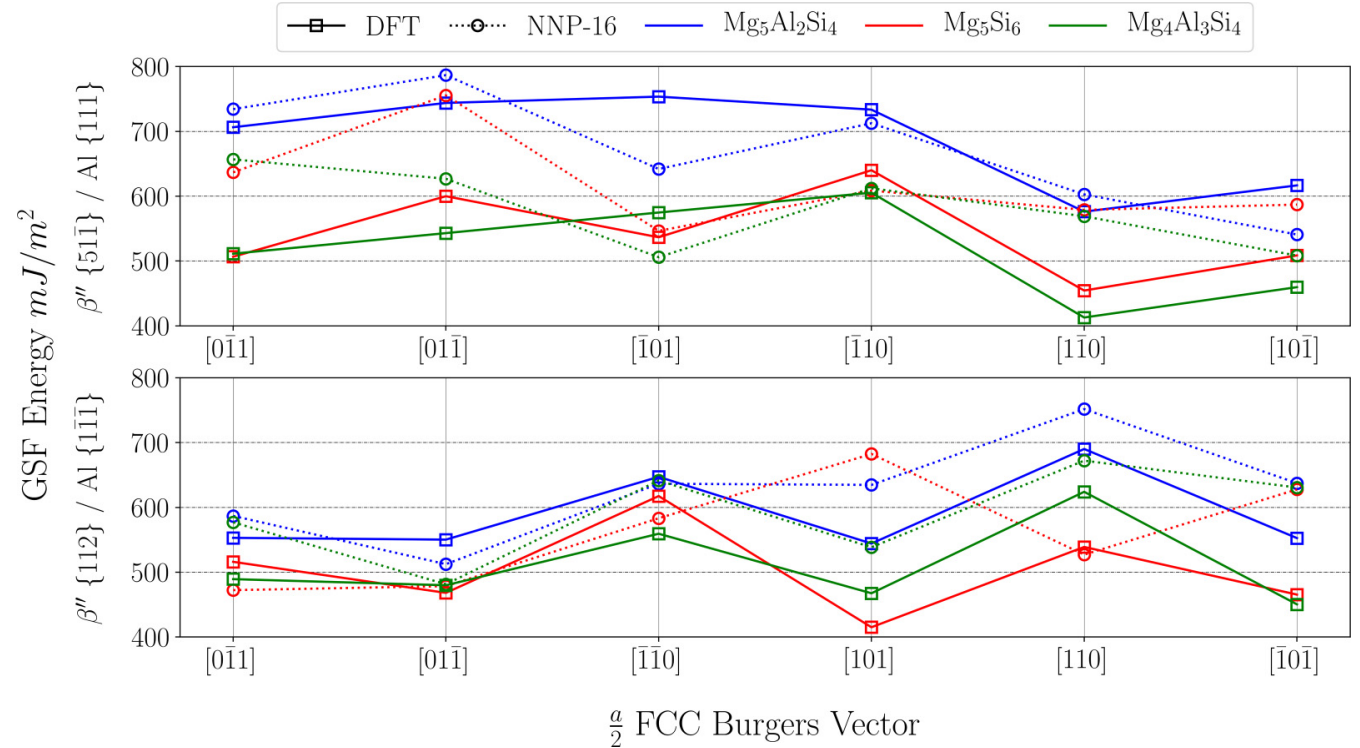

FIG. 1. Burgers vector vs generalized stacking fault energy using neural network potential (NNP-16) and density functional theory for $\mathrm{Mg}_{5} \mathrm{Si}_{6}, \mathrm{Mg}_{5} \mathrm{AlSi}_{4}$, and $\mathrm{Mg}_{4} \mathrm{Al}_{3} \mathrm{Si}_{4}$ in both the $\{51 \overline{1}\}$ and the $\{112\}$ configurations. The NNP shows excellent ability to predict general trends,

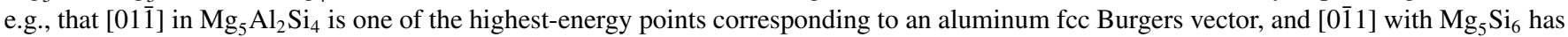
one of the lowest-energy points. These energies do not include the Lennard-Jones (LJ) term, using the LJ term is largely the same with a few points deviating by $10 \mathrm{~mJ} / \mathrm{m}^{2}$. 